\title{
精准纳米气体治疗研究进展
}

\author{
何前军，陈丹阳，范明俭 \\ (深圳大学医学部生物医学工程学院, 深圳 518060)
}

\begin{abstract}
摘 要: 精准纳米气体治疗具有低毒高效等特性, 作为一种新兴的疾病治疗手段受到越来越多的关注。研究表明, 纳米气体治疗不仅能在特定疾病部位选择性杀死癌细胞, 还能保护正常细胞。本文总结了国际最新研究成果, 对精 准纳米气体治疗的最新研究进展进行了总结归纳和展望。首先, 阐述了纳米气体治疗的治疗作用和特点; 然后, 总 结了实现精准纳米气体治疗的主要途径, 包括靶向气体传输、可控气体释放、医学成像引导和监控气体治疗、基于 治疗性气体的多模式联合治疗等; 最后，对纳米气体治疗存在的问题和发展前景做出了总结和展望。
\end{abstract}

关 键 词: 气体治疗; 纳米药物; 可控释放; 药物传输; 介孔二氧化硅; 石墨烯; 综述

中图分类号: TB381 文献标识码: A

\section{Progress of Precision Nanomedicine-mediated Gas Therapy}

\author{
HE Qian-Jun, CHEN Dan-Yang, FAN Ming-Jian
}

(School of Biomedical Engineering, Health Science Center, Shenzhen University, Shenzhen 518060, China)

\begin{abstract}
The precision nanomedicine-mediated gas therapy as an emerging and promising therapy strategy has received increasing attention, due to its low toxicity and high efficacy. Recent studies show that the nanomedicine-mediated gas therapy can not only kill cancer cells in specific diseased sites, but also protect normal cells. This review summarizes and reviews the most recent progress of the nanomedicine-mediated gas therapy. We introduce the therapeutic effects and characteristics of the nanomedicine-mediated gas therapy firstly, and then review several main routes to realize the precision nanomedicine-mediated gas therapy, including targeted gas delivery, controlled gas release, biomedical imaging guidance-monitoring of gas therapy, multi-model combined therapy based on therapeutic gases, and so on, and finally summarize the present existing issues and the prospects of further development in the gas therapy field.
\end{abstract}

Key words: gas therapy; nanomedicine; controlled release; drug delivery; mesoporous silica; graphene; review

生物体内存在一类特殊的气体信号分子, 包括 一氧化氮 $(\mathrm{NO})$ 、一氧化碳 $(\mathrm{CO})$ 和硫化氢 $\left(\mathrm{H}_{2} \mathrm{~S}\right)$ 。这类 气体信号分子可以在与多价过渡金属特异性结合过 程中起到信使的作用, 从而具有调节神经系统、心 血管系统和免疫系统等几乎所有人体系统的多种生 理功能, 对人体生理过程的正常运转和病理过程的
积极调控具有重要作用 ${ }^{[1-5]}$ 。内源性气体信号分子具 有维持体内生理平衡的特殊作用，在体内是不可或 缺的重要物质, 气体信号通路的异常变化往往反映 了疾病的发生与发展 ${ }^{[6-8]}$ 。外源性气体补充对众多重 大疾病具有显著的治疗作用，譬如心血管疾病、癌 症、缺血性损伤、炎症和中风等 ${ }^{[9-14]}$ 。Furchgott、

收稿日期: 2017-11-09; 收到修改稿日期：2017-12-27

基金项目: 国家自然科学基金(81701827); 深圳市基础研究项目(JCYJ20170302151858466); 深圳市孔雀团队项目 (KQTD2016053112051497)

National Natural Science Foundation of China (81701827); Shenzhen Basic Research Project (JCYJ20170302151858466); Shenzhen Peacock Plan (KQTD2016053112051497)

作者简介: 何前军(1979-), 男, 教授. E-mail: nanoflower@126.com 
Ignarro 和 Mura 发现 NO 就是心血管系统的信号分 子, 他们还用实验阐释了 $\mathrm{NO}$ 前体药物分子在心血 管疾病治疗中作用的机制，获得了 1998 年 Nobel 生 理与医学奖 ${ }^{[15]}$ 。这类治疗性气体分子还具有选择性 抗癌的特殊性质：它们不仅可以通过快速耗尽癌细 胞的高水平生物能(即抗沃伯格效应)加速癌细胞的 调亡, 阻止癌细胞的增殖, 还能维持正常细胞的能 量水平, 选择性地保护正常细胞的活性和生理功 能 ${ }^{[16-18]}$ 。这种奇特的肿瘤细胞选择性不仅避免了气 体药物对正常组织的毒性, 而且保护正常细胞, 具 有低毒、高效的抗癌特性, 显著优于目前常用的抗 肿瘤化疗药物。因而, 气体治疗成为一种新兴的、 且非常有应用前景的抗癌治疗策略。目前国内外针 对重大疾病的气体治疗研究正方兴未艾 ${ }^{[19]}$ 。

气体治疗与气体浓度高度相关。众所周知, NO、 $\mathrm{CO}$ 和 $\mathrm{H}_{2} \mathrm{~S}$ 气体在血液中浓度过高都会引起中 毒 $^{[20-22]}$, 但在相对较低的浓度范围内, 它们不仅不 会引起中毒, 反而具有显著的抗癌活性。理想的安 全且有效的气体治疗策略是：既保证治疗性气体特 异性高浓度蓄积于病灶部位, 又保证治疗性气体在 血液中保持较低的安全浓度 ${ }^{[19]}$ 。临床上治疗性气体 主要有两种给药方式: 直接吸入气体、口服或腹部 注射气体释放分子(Gas-Releasing Molecules, GRMs) 药物。这两种给药方式都无法实现靶向性气体运输 和可控气体释放, 主要归因于这些气体分子在体内 具有快速自由扩散的行为 ${ }^{[1]}$ 。

随着纳米技术的飞速发展，大量新型多功能纳 米药物载体材料被研发出来, 这为通过构建纳米药 物来实现药物的靶向传输与可控释放提供了条 件 ${ }^{[23-26]}$ 。本课题组开创性地提出精准纳米气体治疗 的概念来实现疾病的精准治疗 ${ }^{[19]}$ 。纳米气体治疗是 指借助纳米技术将多功能纳米载体与气体或能产生 治疗性气体的前驱体药物 GRMs 进行组合, 构建出 一类智能纳米气体药物, 从而成为疾病精准治疗的 一种途径。精准纳米气体治疗可以通过靶向气体传 输、可控气体释放、医学成像引导和监控气体治疗、 基于治疗性气体的多模式联合治疗等途径来实现高 效、低毒的疾病治疗, 对于发展气体治疗用于重大 疾病的研究具有重要的科学意义。本文将从上述几 个方面综述近年来的进展。

\section{1 可控气体释放}

从纳米药物结构的角度考虑, 本文提出两种策 略来实现可控气体释放: 1)开发刺激敏感性 GRMs 用于构建响应性纳米药物; 2) 通过纳米载体与
GRMs 之间的协同作用实现气体的可控释放 ${ }^{[19]}$ 。这 种协同作用可以来源于纳米载体与 GRMs 之间的能

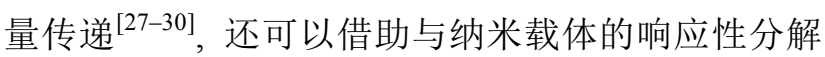
或结构破裂 ${ }^{[31-33]}$ 。从刺激源的角度考虑, 两类策略 可以实现可控气体释放: 1)外源性刺激响应, 如光、 声、电和磁等; 2)内源性刺激响应, 如酸碱性、氧化 还原性、过度表达的双氧水水平、ATP 水平和特殊 酶水平等。外刺激源响应具有刺激源容易操控的优 势, 包括刺激源的开关、聚焦、能量调节和辐照时 间的调节等; 内刺激源响应具有组织渗透深度没有 限制的优势, 特别适合用于常规药物无法渗透到的 疾病组织部位, 如肿瘤组织内部等。

\section{1 外刺激源响应性可控气体释放 \\ 1.1.1 近红外光控制气体释放}

在众多外源性刺激源中, 激光是最方便有效的, 因此光控药物释放成为一种常见的可控给药途径。 大多数光响应性 GRMs 前药只对紫外光或可见光敏

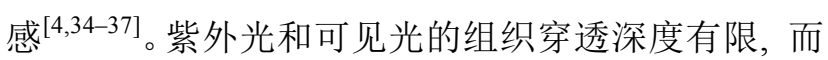
且容易引起光毒性, 严重限制了光响应性气体释放 在体内的应用。相比而言, 近红外(NIR)光具有更大 的组织穿透深度和更低的光毒性, 因而 NIR 光响应 性气体释放具有更广阔的应用前景。根据上述从纳 米药物结构角度提出的两种策略, 本实验室设计合 成了一类对 NIR 光敏感的 GRMs ${ }^{[38]}$, 通过氧化石墨 烯纳米片与 UV 敏感性 GRMs 间的光电转化效应, 实现了 NIR 光响应性 $\mathrm{CO} / \mathrm{NO}$ 气体可控释放。

2015 年, 本课题组使用超小氧化石墨烯纳米片 (Graphene Oxide Nanosheet, GON)与金属羰基化合 物(羰基锰, $\mathrm{MnCO}$ )作为纳米载体和能产生 $\mathrm{CO}$ 气体 的前药(CO-Releasing Molecules, CORMs), 通过络 合作用构建了一种“笼状”纳米药物 $\mathrm{MnCO}-\mathrm{GON}^{[27]}$ (图 1(A))。通过氧化石墨烯的 NIR 光吸收效应、光 电转化效应和光一化学能转化原理, 吸收 NIR 光能, 从而激发表面铆接的 CORMs 前药化学分解, 释放 $\mathrm{CO}$, 从而实现了 NIR 光响应性释放 $\mathrm{CO}$ 气体。实验 结果表明: $\operatorname{MnBr}(\mathrm{CO})_{5}$ 前药只对 $\mathrm{UV}$ 光敏感, 而对 $\mathrm{NIR}$ 光不敏感, 然而构建的 $\mathrm{MnCO}-\mathrm{GON}$ 纳米药物 变得对 NIR 光敏感; $\mathrm{MnCO}-\mathrm{GON}$ 纳米药物的 NIR 光 响应性 $\mathrm{CO}$ 释放依赖于 NIR 光的能量密度和辐照时 间(图 1(B))。越高的 NIR 光能量导致 CO 释放越快, 越长的 NIR 光辐照时间导致 CO 释放越多。除了具 有高 NIR 光敏感性, MnCO-GON 纳米药物还具有高 NIR 光可控性。开启 NIR 光源导致 CO 快速释放, 而 关掉 NIR 光源迅速导致 $\mathrm{CO}$ 释放中止, 循环开关周 而复始(图 1(C))。这表明构建的 MnCO-GON 纳米药 
物可以实现按需可控的 $\mathrm{CO}$ 释放。通过 MnCO-GON 纳米药物介导的细胞内药物传输和胞内 NIR 光控 $\mathrm{CO}$ 释放, 实现了胞内 $\mathrm{CO}$ 的可控释放和蓄积, 而且 胞内释放的 CO 表现出明显的抗炎作用。借助类似 的原理, 本实验室通过 $\pi-\pi$ 共轭效应, 使用氧化石 墨烯纳米片和 bis- $N$-nitroso(BNN)型 $\mathrm{NO}$ 气体释放分 子(NO-Releasing Molecules, NORMs)层层堆叠, 构 建了一种新型的 “三明治”纳米结构 GON-BNN6 (图 2(A))。同样利用 GON 的 NIR 光吸收效应和光 电转化效应(图 2(B)), 实现了 NIR 光响应性的 NO 释放(图 2(C) $)^{[28]}$ 。此外, 赵东元和赵宇亮课题组 ${ }^{[29-30]}$ 将 UV 光敏感的罗森黑盐(RBS)型 NORMs 前药装载 到上转换纳米颗粒@介孔二氧化硅纳米颗粒的介孔 壳中, 通过上转换纳米核的 NIR-to-UV/Vis 上转换 特性, 吸收 NIR 光, 发射 UV 光, 激发壳层中的 NORMs 前药, 释放 NO 气体, 从而实现了 NIR 响应 性释放 NO 气体。上述实验表明, 通过调节 NIR 光
开关、辐射时间和能量密度可以较好地控制纳米药 物的气体释放, 从而达到治疗目的。

最近，本课题组采用一种简便的配位络合一沉 淀法，直接合成了一类对 NIR 光敏感的新型 $\mathrm{GRMs}^{[38]}$ 。RBS 是一种对 $\mathrm{UV}$ 光敏感的可溶性 NORMs, 因而具有较大的细胞毒性和热不稳定性。 因此，可通过配位络合-沉淀途径，通过过渡金属离 子将 RBS 阴离子沉淀下来, 合成一种难溶的、热稳 定的、对 NIR 光敏感的新型罗森黑盐(Me-RBS)。所 用合成 Me-RBS 的配位络合-沉淀法极为简便、快 捷、普适性强，只需将金属离子溶液和 RBS 溶液混 合、沉淀、分离, 即可得到 Me-RBS 产物(图 3(C)), 适 用的金属离子产量都比较高(一般 $>90 \%$ )。Me-RBS 的形成与过渡金属阳离子跟 RBS 阴离子间的配位 作用密切相关, 研究结果表明铜、铁、铅、钴和铝 离子能够与 RBS 形成 Me-RBS 化合物。Me-RBS 化 合物的 UV-Vis-NIR 吸收行为与过渡金属离子的性
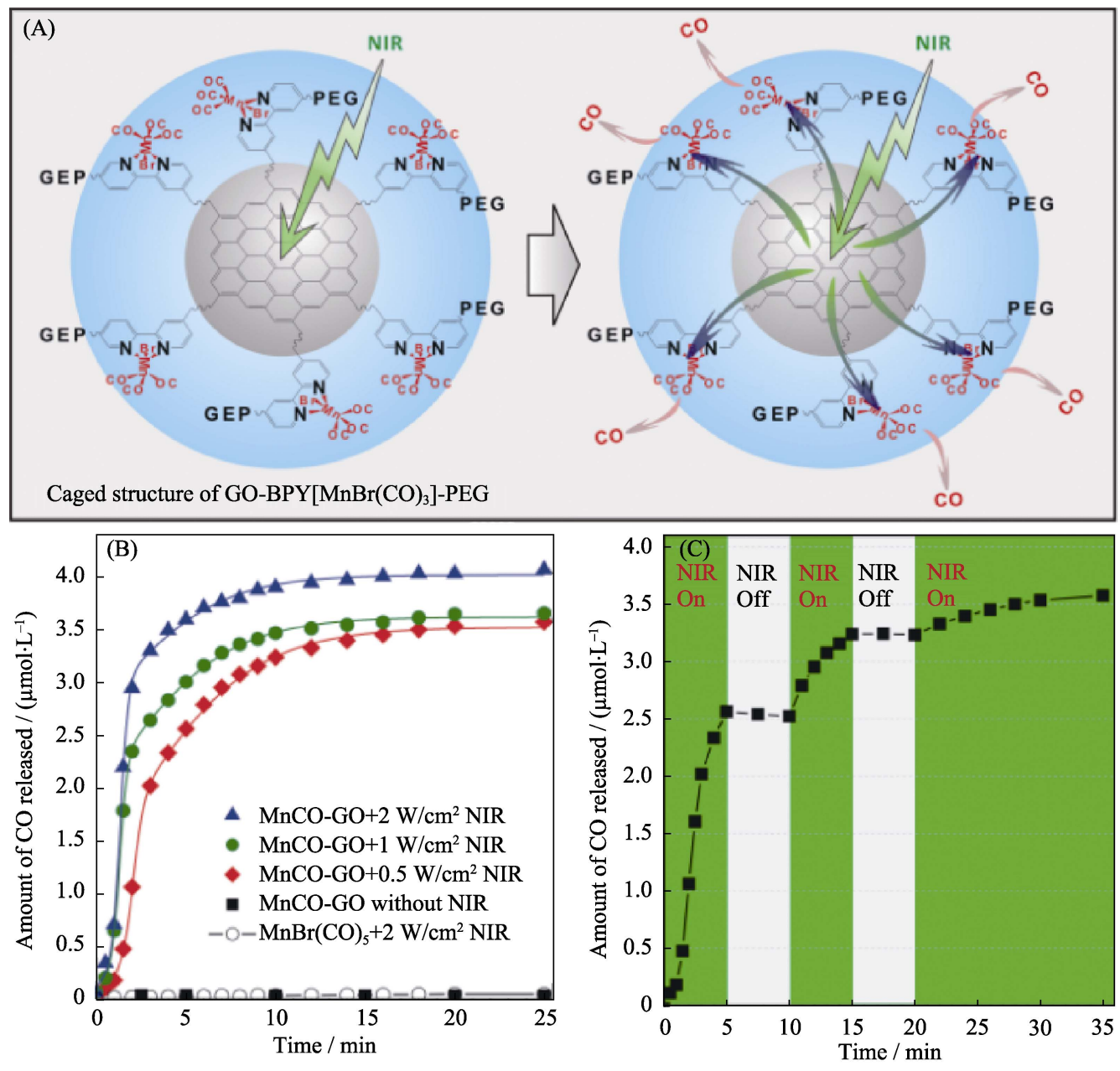

图 1 (A)具有“笼状”结构 $\mathrm{MnCO}-\mathrm{GON}$ 纳米药物的 NIR 光响应性释放 $\mathrm{CO}$ 气体的原理示意图,

(B)纳米药物可控释放 CO 的 NIR 光响应性和(C)纳米药物可控释放 CO 的 NIR 光可控性 [27]

Fig. 1 (A) NIR-responsive CO release mechanism of the MnCO-GON nanomedicine with a caged structure,

(B) NIR responsive for CO release profiles of MnCO-GON, and (C) NIR-controllability of MnCO-GON for CO release ${ }^{[27]}$ GON: Graphene Oxide Nanosheet 

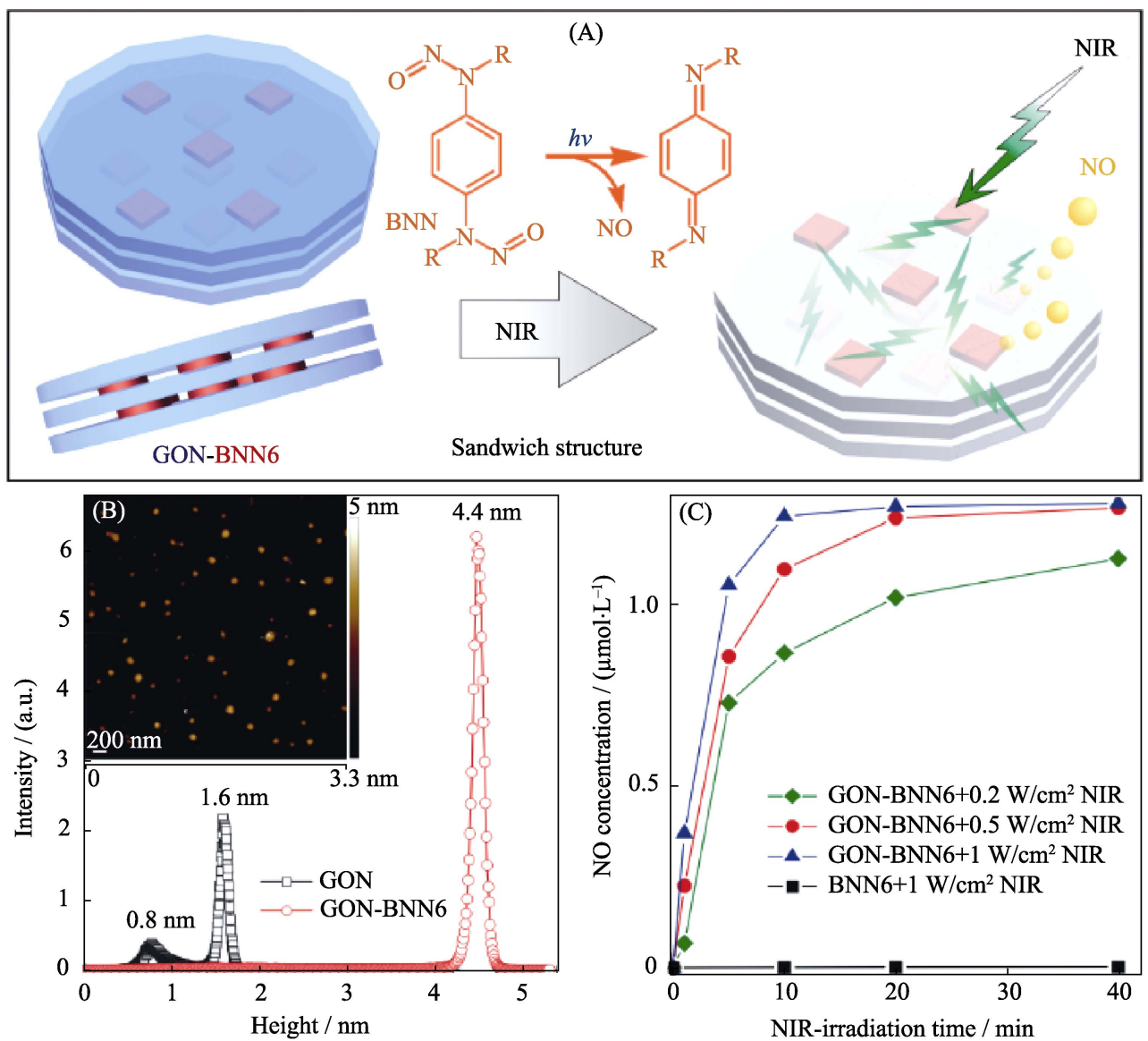

图 2 (A)具有 “三明治” 结构的 GON-BNN6 纳米药物的 $\pi-\pi$ 共轭自组装, 及其 NIR 光响应性释放 NO 气体的原理示意图, (B)纳米药物的 AFM 形貌表征和(C)纳米药物的 NIR 可控释放 NO 行为 ${ }^{[28]}$

Fig. 2 (A) The sandwich structure of GO-BNN6 self-assembled by GO nanosheets and BNN6 molecules through the $\pi-\pi$ stacking, and the mechanism of NIR-responsive NO release; (B) AFM data of GO-BNN6 and (C) NIR-controlled NO release profiles of the GO-BNN6 nanomedicine ${ }^{[28]}$ BNN: bis- $N$-nitroso

质密切相关, Me-RBS 的 NIR 光响应性又与其对 NIR 光的吸收密切相关(图 3(D)和(E))。RBS 在 NIR 区域 无吸收, 因而对 NIR 光不敏感; Cu-RBS 的 NIR 光吸 收最强, 因而其对 NIR 光最敏感, NIR 光响应性释 放 NO 的速度最快。因此, 通过分子结构设计改变 其光学性质进而改变其光控气体释放行为的策略, 对于设计合成新型光敏感性 GRMs 分子化合物具有 重要的指导意义。

\subsection{2 超声控制气体释放}

与光刺激相比, 超声波能够更方便地聚焦于人 体组织内部的局部区域 (切向焦点尺寸可达微米 级)、具有更强的组织穿透性(1 MHz 声波的组织穿 透深度可达 $20 \mathrm{~cm}$ ), 因而超声响应性药物控释成为 一种独具特色和优势的给药方式 ${ }^{[39]}$ 。利用超声波刺 激药物, 进行响应性地可控释放治疗性气体的研究 目前只集中在包裹气体的脂质体型纳米药物, 即采 用脂质体微泡包裹气体分子构建的纳米药物, 通过 超声波的空化作用破碎脂质体微泡, 从而释放其中
包裹的气体分子。然而目前这种超声微泡型纳米药 物存在的缺点主要包括气体担载量较低、稳定性差、 无法达到可控和大小颗粒不均一等 ${ }^{[40]}$ 。无机载体如 中空介孔二氧化硅纳米颗粒(hMSN) 比有机脂质体 载体物理化学稳定性高得多, GRM@hMSN 纳米医 药可以克服传统气体@脂质体纳米药物的上述缺点, 有利于避免药物泄漏等。因此开发性能优异的新型 超声响应性纳米药物, 并实现治疗性气体在体内安 全可控释放, 是气体治疗当前亟待解决的关键科学 问题。

最近, 本课题组首次发现光响应性 BNN6 药物 也对超声具有响应性，超声波能加速 NO 自由基分 子的产生, 从而触发 BNN6 药物分解释放 NO 气体 (图 4(A) $)^{[41]}$ 。BNN6 是一种高度疏水的气体前药, 因 此为了改善药物的传输效率, 本课题组设计了一种 铃铛型结构的空心介孔二氧化硅(SPION@hMSN) 用于 BNN6 药物的高效装载与靶向传输(图 4(B))。所 构建的纳米药物 BNN6-SPION@hMSN 对超声波具 

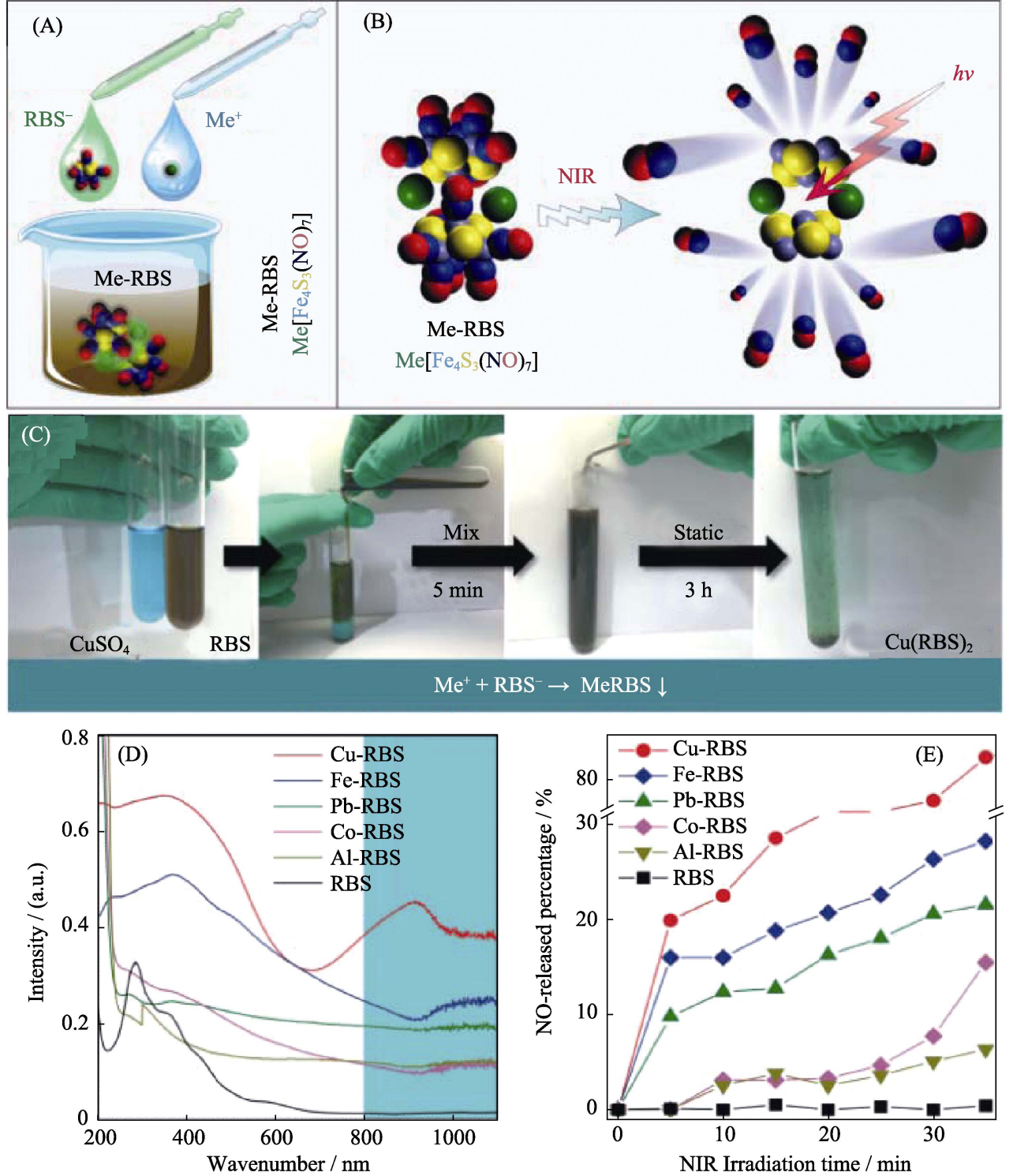

图 3 (A)不溶性金属配位型罗森黑盐 Me-RBS 的配位络合-沉淀法示意图; (B)Me-RBS 的 NIR 光响应性释放 $\mathrm{NO}$ 气体的原理示意图; (C)Me-RBS 的紫外吸收行为比较和(D)NIR 光控释放 NO 行为比较 ${ }^{[38]}$

Fig. 3 (A) Schematic illustration of the coordination-precipitation process of insoluble Me-RBS,

(B) the NIR-responsive NO release mechanism of Me-RBS, (C) comparison of UV absorption behaviors of Me-RBS and (D) comparison of NO release behaviors of Me-RBS ${ }^{[38]}$

RBS: Rosen Black Salt

有较高的响应性, NO 释放速度及其细胞毒性与超声 波能量正向相关(图 4(C)和(D))。相比于传统的气体@ 脂质体纳米药物, 利用空心介孔二氧化硅载体和气体 前药设计的新型超声响应性纳米药物 GRM@hMSN, 充分体现了载气量大、稳定性好、可控性强、粒径 均一等优点, 具有更高的应用价值。

\subsection{3 $\mathrm{X}$ 射线控制气体释放}

作为一种非侵入性的外源性刺激, $\mathrm{X}$ 射线在活 体的穿透率极高, 并且低剂量的 $\mathrm{X}$ 射线辐射可以潜 在地在空间和时间上触发药物的释放。 $X$ 射线在控 制药物释放方面具有以下优势: 首先, 高度穿透的 $\mathrm{X}$ 射线可以在深部病变区域进行定点控制药物释放;
其次, 通过改变 $\mathrm{X}$ 射线辐射剂量和持续时间, 可实 现按需药物释放; 最后, 在肿瘤治疗中运用 $\mathrm{X}$ 射线 触发药物释放, 将化疗与放疗结合, 从而提高治疗 效果。实验证明, 在肿瘤放射治疗中, $\mathrm{NO}$ 气体/自由 基可以为放疗增敏。相对较高浓度的 NO 可作为一 种有效的低氧放射增敏剂来增强放射疗效, 但过高 的 NO 可能带来潜在的中毒风险。因此, 本课题组 使用一种核壳结构的介孔二氧化硅载体(Upconversion nano-theranostic system, PEG-USMSs)担载 S亚硝基硫醇(S-nitrosothiol, SNO)型 NORMs 前药,设 计合成了一种新型介孔基纳米药物(PEG-USMSs$\mathrm{SNO}$ ), 担载的 SNO 前药响应 X 射线可控释放 NO, 

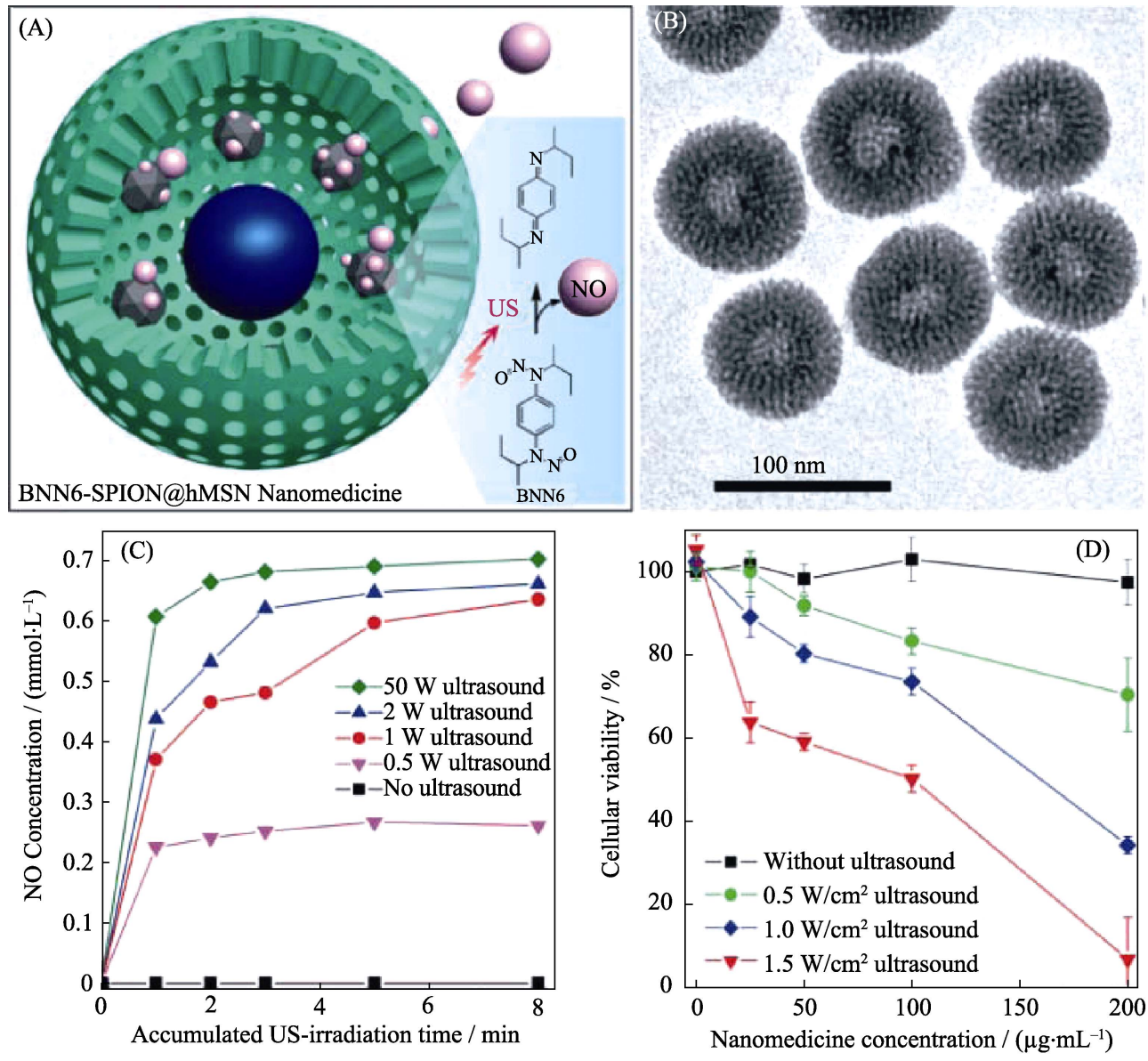

图 4 (A)具有“铃铛”结构的 BNN6-SPION@hMSN 纳米药物的超声响应释放 NO 气体的原理示意图, (B)纳米药物的 TEM 照片, (C)纳米药物的超声可控释放 NO 行为和(D)超声诱导纳米药物细胞毒性行为 ${ }^{[41]}$

Fig. 4 (A) The mechanism of ultrasound-responsive NO release from the rattle-structured BNN6-SPION@hMSN nanomedicine, (B) TEM image of the nanomedicine, (C) ultrasound-responsive NO release behavior of the nanomedicine and (D) ultrasound-induced cytotoxicity of the nanomedicine ${ }^{[41]}$

BNN: bis- $N$-nitroso; SPION: Superparamagnetic Iron Oxide-encapsulated; hMSN: hollow Mesoporous Silica Nanoparticles

实现 NO 气体治疗和放疗的联合增效(图 5) ${ }^{[42]}$ 。 PEG-USMSs-SNO 纳米药物在体外和动物体内都表 现出了良好的 X 射线响应性(图 5(A)、(B)), X 射线 剂量越高, $\mathrm{NO}$ 的释放量越大。但是该体系的缺点在 于 $\mathrm{NO}$ 释放的可控性较差，在无 X 射线辐照的情况 下 NO 依然会缓慢地自发释放, 这主要起因于 SNO 前药在生理环境下的不稳定性。因此, 如何改善 X 射线响应性纳米药物的稳定性和 X 射线控释 NO 的 可控性, 是今后需要解决的一个重要问题。

\section{2 内刺激源响应性可控气体释放}

内刺激源响应性可控气体释放主要是利用机体 内病变部位特殊的微环境, 如肿瘤微环境(包括肿 瘤内酸性环境, 还原性环境和过氧化氢过表达环境 等), 纳米药物在该微环境的刺激下释放气体。相对 于光刺激和声刺激等外源性刺激源而言，内源性刺 激是一种无创、绿色的介导气体释放的方式，具有 在体内穿透的深度不受限制、在不侵袭正常组织和 细胞的情况下实现原位靶向释放的优点。

\subsection{1 双氧水响应性气体释放}

肿瘤细胞中的 $\mathrm{H}_{2} \mathrm{O}_{2}$ 水平明显高于正常细胞, 约 为正常细胞的 3 26倍, 因此可利用肿瘤细胞中的高 $\mathrm{H}_{2} \mathrm{O}_{2}$ 表达量设计一类双氧水响应性纳米药物。 $\mathrm{H}_{2} \mathrm{O}_{2}$ 响应性气体释放主要是运用肿瘤内的 $\mathrm{H}_{2} \mathrm{O}_{2}$ 触发纳 米药物释放治疗性气体, 从而原位杀死肿瘤细胞。 实现双氧水响应性气体释放的一个主要途径是开发 双氧水响应的气体前药。本课题组最近发现金属羰 基化合物前药是一种新型的类 Fenton 试剂, 可以催 化双氧水分解产生高氧化活性的羟基自由基, 产生 的羟基自由基氧化金属羰基化合物，从而产生 $\mathrm{CO}$ 气体(图 6(A) $)^{[43]}$ 。但大多数稳定的金属羰基化合物 都是强疏水性药物，因此采用空心介孔二氧化硅高 效担载羰基锰，构建了一种双氧水响应的新型纳米 药物 $\mathrm{MnCO} @ \mathrm{hMSN}$ (图 6(B))。 $\mathrm{H}_{2} \mathrm{O}_{2}$ 触发的 $\mathrm{CO}$ 释 放行为和细胞毒性高度依赖于细胞内双氧水的水 平(图 6(C) (E)), $\mathrm{H}_{2} \mathrm{O}_{2}$ 含量较高的肿瘤细胞触发纳 米药物释放更多的 $\mathrm{CO}$ 从而达到更有效的抗肿瘤 

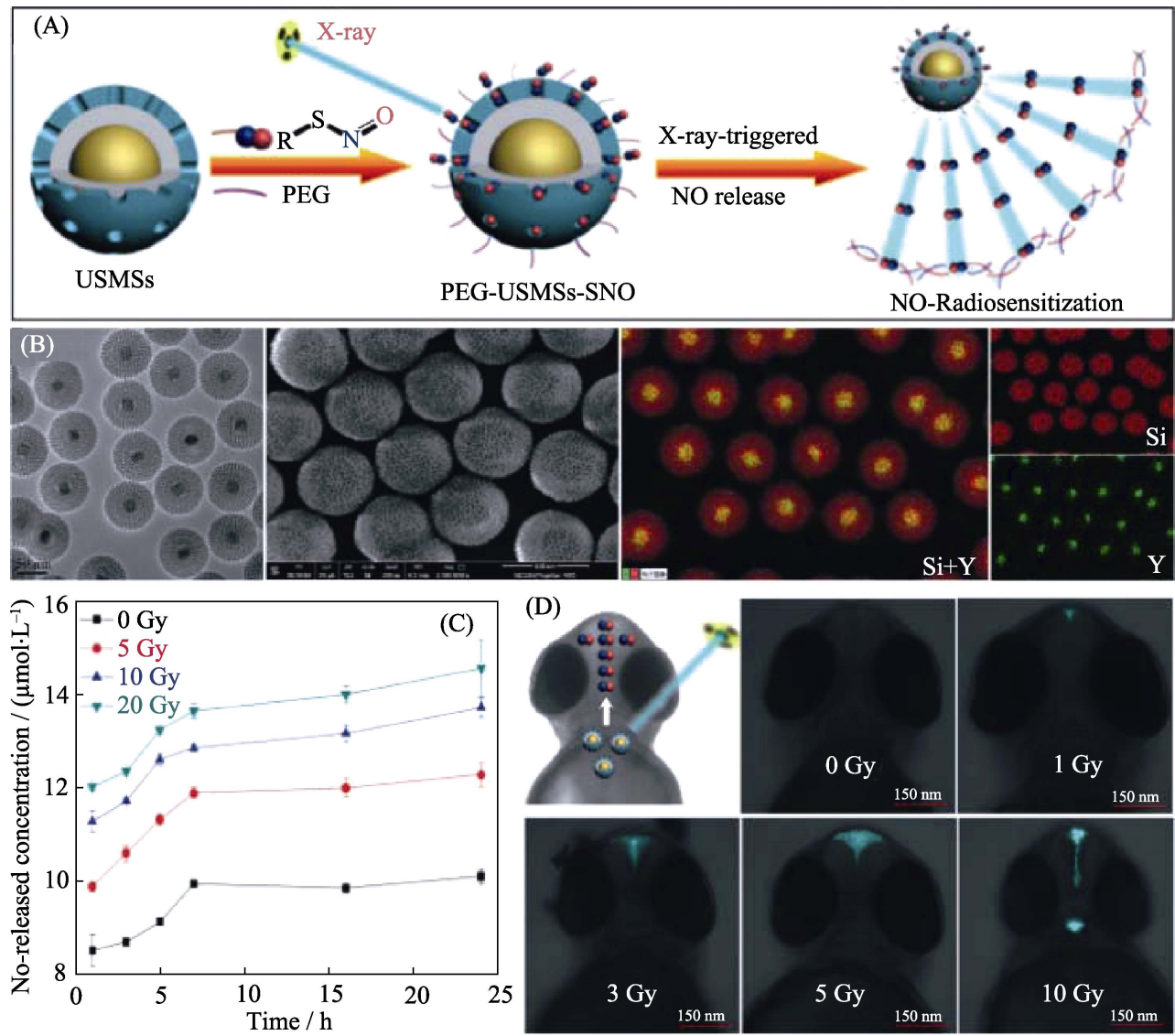

图 5 (A)具有 “核壳” 结构的 PEG-USMSs-SNO 纳米药物的 X 射线响应性释放 NO 气体的原理示意图, (B)纳米药物的 TEM 照 片和元素分布图, (C)纳米药物在体外的 X 射线可控释放 NO 行为和(D)纳米药物在斑马鱼体内的 X 射线可控释放 NO 行为 ${ }^{[42]}$ Fig. 5 (A) The mechanism of X-ray responsive NO release from the PEG-USMSs-SNO nanomedicine with the core-shell structure, (B) TEM images and elementary mapping of the nanomedicine, (C) X-ray controlled NO release behavior of the nanomedicine in vitro, and (D) X-ray controlled NO release behavior of the nanomedicine on zebrafish ${ }^{[42]}$

USMSs: Upconversion nano-theranostic system; SNO: S-nitrosothiol.

治疗。该纳米药物对 $\mathrm{H}_{2} \mathrm{O}_{2}$ 过表达的肿瘤细胞产生较 高的细胞毒性，但对正常细胞几乎没有细胞毒性， 从而表现出较高的选择性抗癌效果。由于这种选择 性的杀伤肿瘤作用和通过纳米药物对 $\mathrm{CO}$ 气体的肿 瘤位点特异性递送性质, 已经证明了体外和体内癌 症治疗的高效性和良好的生物安全性。

\subsection{2 葡萄糖响应性气体释放}

葡萄糖是肿瘤生长的主要营养物质和能量来 源。肿瘤对葡萄糖的需求远高于正常组织, 因而葡萄 糖也是一种潜在的内源性刺激源。本课题组以空心 介孔有机硅 $(\mathrm{hMON})$ 作为载体, 高效负载葡萄糖氧 化酶(GOx)和 L-精氨酸(L-Arg), 构建了一种新型葡 萄糖响应性纳米药物 Arg@hMON-GOx (图 7(A) $)^{[44]}$ 。 GOx 催化葡萄糖氧化分解产生葡萄糖酸和双氧水, 产生的双氧水进一步氧化 L-Arg 产生 NO 气体, 从 而实现葡萄糖响应性释放 NO (图 7(B))。研究结果 表明: $\mathrm{pH}$ 、双氧水浓度和 $\mathrm{NO}$ 浓度都与葡萄糖浓度 正向相关; 细胞内的葡萄糖浓度越高, 产生的 $\mathrm{NO}$ 浓度则越高, 抗肿瘤效果越显著。该纳米药物的优 点在于前药担载量高、NO 释放的响应性高、体系
降解性好和生物相容性好; 缺点在于 GOx 的催化反 应无法终止, 且反应速度难以调节, 可控性较差。但 葡萄糖响应性控释气体用于疾病治疗的概念具有潜 在的应用价值，设计开发性能更优异的新型葡萄糖 响应性纳米药物值得进一步研究。

\subsection{3 酸响应性气体释放}

肿瘤和炎症组织的微酸性环境为酸响应性可控 气体释放提供了内源性动力。 $\mathrm{Kim}$ 等 ${ }^{[32]}$ 采用一种酸 可降解载体 $\mathrm{MSN}-\mathrm{CaP}$ 来装载 $\mathrm{NO}$ 气体前药, 实现酸 响应性 $\mathrm{NO}$ 气体释放(图 8(A))。磷酸钙 $\mathrm{CaP}$ 涂层 可以起到保护被装载前药和酸响应性溶解的目的。 在较低 $\mathrm{pH}$ 下, $\mathrm{CaP}$ 涂层溶解, 被装载的 $\mathrm{NO}$ 前药 2-nitrobenzaldehyde(NBA)被暴露出来而自发分解产 生 NO 气体(图 8(B))。由此可见, 极不稳定的 NBA 前药有可能致使该纳米药物不稳定(图 $8(\mathrm{C})$ )。此外, 作者还装载光响应性产酸剂 diazeniumdiolates, 用 紫外光激发其产酸, 用于原位打开 $\mathrm{CaP}$ 壳层(图 8(A))。但该光响应产酸剂仅对紫外光响应, 而且装 载的 NO 前药 NBA 也对紫外光响应释放 NO, 给上 述酸响应控释 NO 的结论造成了困扰。因此, 如何 
既利用微弱的酸性微环境实现酸可控气体释放, 又 保证纳米气体药物在正常生理环境下的高度稳定和 药物无泄漏, 依然是今后亟待解决的关键问题之一。

\section{2 靶向气体运输}

为了消除气体快速自由扩散的缺点, 实现靶向 气体传输至关重要。通过引入靶向性纳米载体来负 载气体前药, 可构建一类新型靶向性纳米气体药物,
从而实现气体的靶向运输。纳米药物的靶向性主要 可以通过两种方式实现：被动靶向和主动靶向。被 动靶向指尺寸在 $100 \mathrm{~nm}$ 以下的颗粒可以通过肿瘤 的“增强渗透与滞留”效应(EPR 效应), 实现纳米颗 粒在肿瘤部位的被动蓄积。因而, 为了实现肿瘤靶 向气体运输, 纳米药物的尺寸应该控制在 $100 \mathrm{~nm}$ 以 下。主动靶向主要通过配体识别(如抗 HER/CD20/ CD40 单抗、靶向肽、叶酸/半乳糖/转铁蛋白)和外场 引导(如磁场靶向) 实现 ${ }^{[45-47]}$ 。Veres 等 ${ }^{[4]}$ 构建了一种
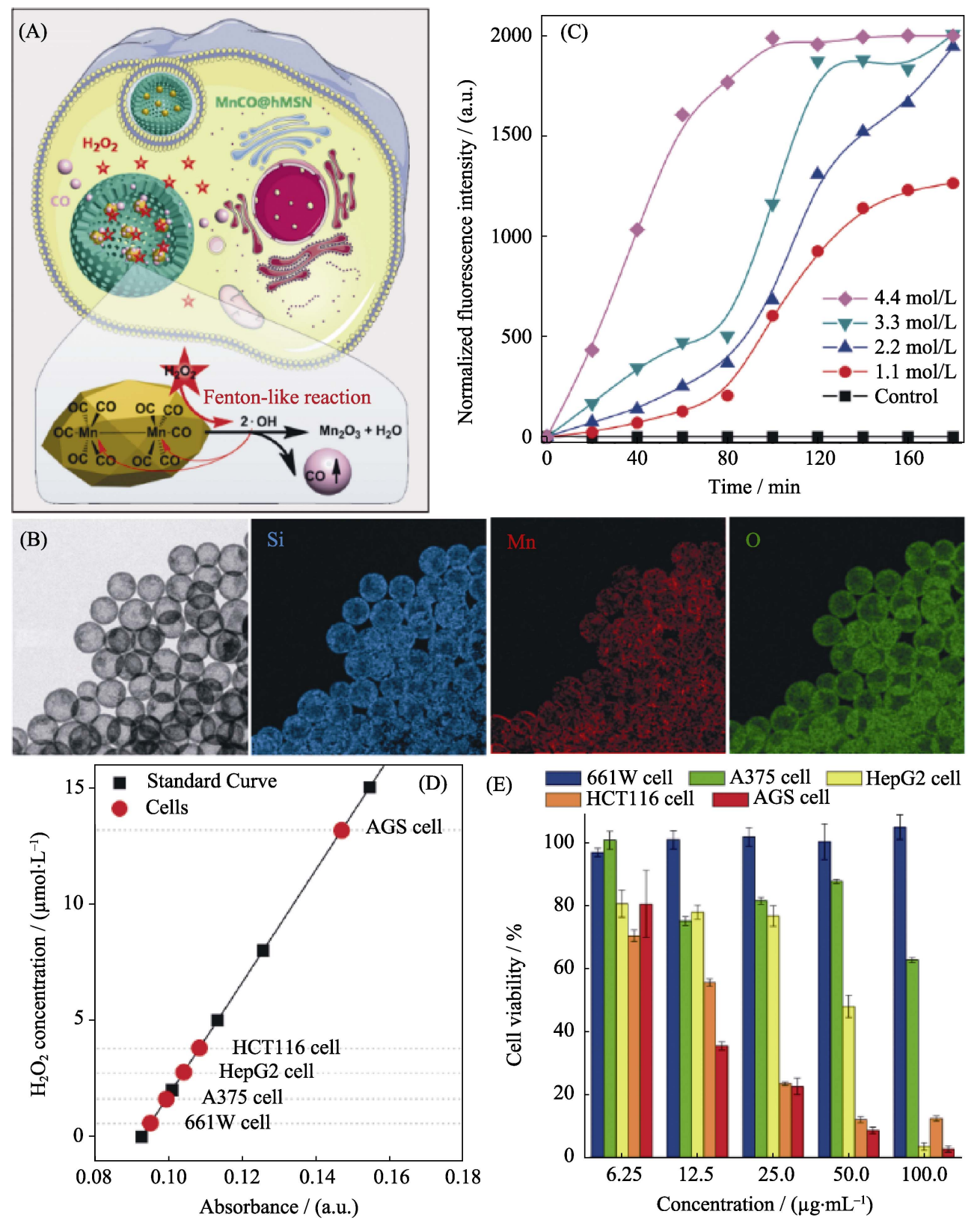

图 6 (A)基于空心介孔二氧化硅纳米载体和羰基锰前药的 $\mathrm{MnCO} @ \mathrm{hMSN}$ 纳米药物的双氧水响应性释放 $\mathrm{CO}$ 气体的原理示意图, (B)纳米药物的 TEM 照片和元素分布图, (C)纳米药物在体外的双氧水可控释放 $\mathrm{CO}$ 行为,

(D)各种细胞内的双氧水水平比较和(E)纳米药物对各种细胞的细胞毒性研究 ${ }^{[43]}$

Fig. 6 (A) The $\mathrm{H}_{2} \mathrm{O}_{2}$-triggered CO release mechanism of the MnCO@hMSN nanomedicine constructed by hMSN and manganese carbonyl prodrug, (B) TEM image and elementary mapping of the nanomedicine, (C) $\mathrm{H}_{2} \mathrm{O}_{2}$-triggered CO

release behavior of the nanomedicine in vitro, (D) comparison of $\mathrm{H}_{2} \mathrm{O}_{2}$ levels in various cells and

(E) comparison of cytotoxicity against various cells ${ }^{[43]}$ hMSN: hollow Mesoporous Silica Nanoparticles 

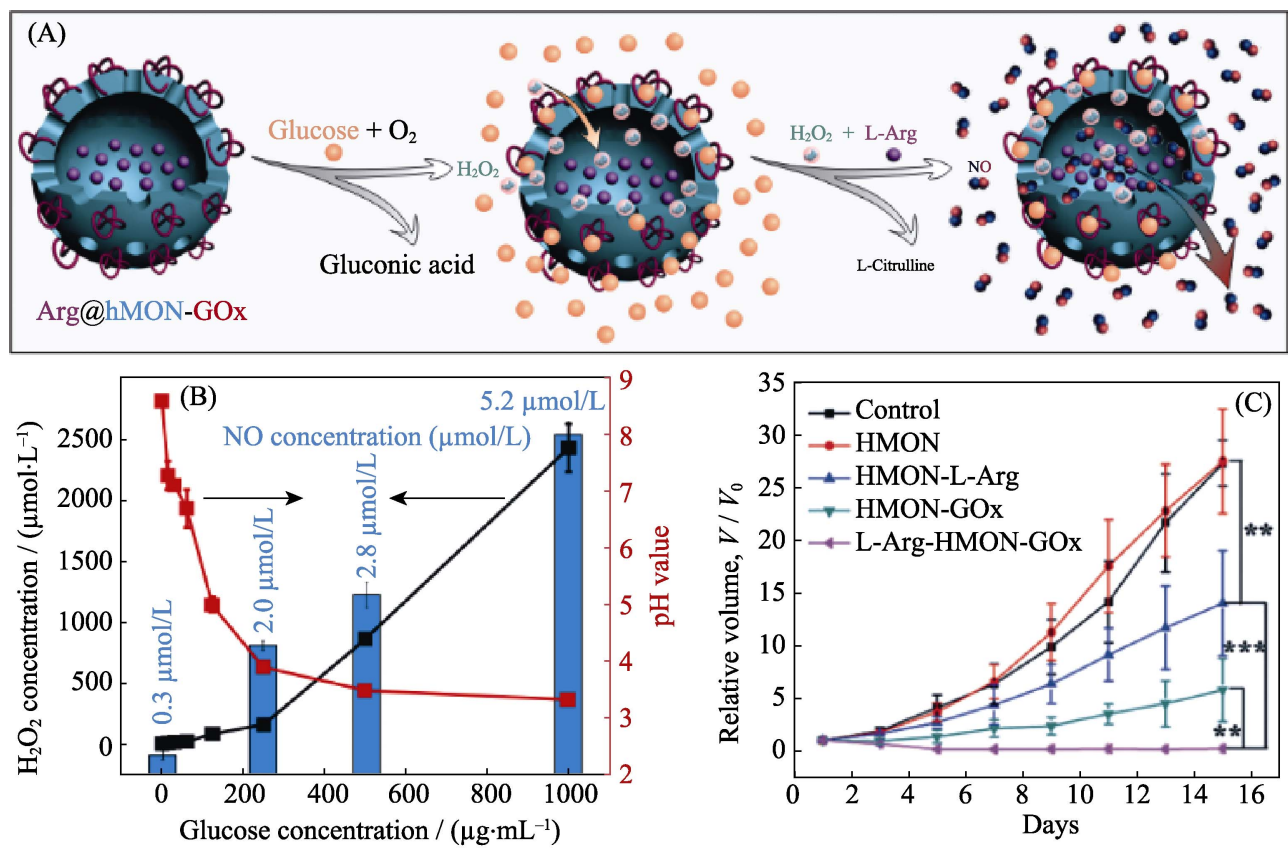

图 7 (A)基于空心介孔二氧化硅纳米载体和葡萄糖氧化酶/L-精氨酸前药的 Arg@hMON-GOx 纳米药物的构建及其葡萄糖响 应性释放 $\mathrm{NO}$ 气体的原理示意图, (B)葡萄糖浓度对双氧水浓度、 $\mathrm{pH}$ 和 $\mathrm{NO}$ 浓度的影响和 $(\mathrm{C})$ 纳米药物对荷瘤鼠的治疗作用 ${ }^{[44]}$

Fig. 7 (A) Construction and SEM image of the Arg@hMON-GOx nanomedicine based on the hMON carrier and the Arginie/GOx prodrugs, and the mechanism of glucose-responsive release of NO, (B) effects of glucose concentration on hydrogen peroxide concentration, $\mathrm{pH}$ and $\mathrm{NO}$ concentration, and $(\mathrm{C})$ in vivo outcome of gas therapy by nanomedicine $\mathrm{e}^{[44]}$ hMON: hollow Mesoporous Organosilica Nanoparticle

磁性四氧化铁-NORMs-二氧化硅纳米颗粒, 通过磁 靶向策略实现了纳米颗粒在体外模拟条件下的磁引 导蓄积, 但较难在动物体内实现精确的三维磁定位 靶向。本课题组借助 EPR 效应, 构建具有较小尺寸 (80 nm) 的纳米气体药物 BNN6- SPION@hMSN 实 现了肿瘤内的高效被动靶向药物传输(图 9(A) $9(\mathrm{~B}))^{[41]}$ 。目前用于气体治疗的靶向性纳米药物的报 道依然较少。

\section{3 医学成像引导气体治疗}

除了靶向气体传输, 本课题组还提出利用医学 成像引导气体治疗, 也是实现精准气体治疗的一个 有效途径 ${ }^{[19]}$ 。如何通过有效整合多功能纳米载体和 气体前药, 构建兼具医学成像功能和气体控释功能 的纳米气体药物, 是实现医学成像引导气体治疗的 关键。基于这一思路, 本课题组首次提出构建超顺 磁性空心介孔二氧化硅纳米载体 SPION@hMSN, 用于高效装载气体前药 BNN6, 也用于磁共振成像 (Magnetic Resonance Imaging, MRI)引导超声控释 $\mathrm{NO}$, 实现 $\mathrm{NO}$ 气体精准治疗 ${ }^{[41]}$ 。10 nm 的 SPION 核 赋予了纳米药物较高的超顺磁性和较高的 MRI 成 像性能; 整个纳米药物 BNN6-SPION@hMSN 的颗 粒尺寸仅 $80 \mathrm{~nm}$, 使其具有较好的肿瘤被动靶向性
能。荷瘤鼠实验结果表明：尾静脉注射 BNN6SPION@hMSN 纳米药物后, 在 $8 \mathrm{~h}$ 内逐渐在肿瘤内 靶向蓄积(图 9(B)); 通过 MRI 成像可准确地定位肿 瘤位置(图 9(B)箭头所示), 为针对肿瘤部位聚焦超声 触发纳米药物原位释放 NO 提供了条件。这种医学成 像引导气体治疗的途径极大地提高了气体治疗的精 准性和有效性，不仅可以有效地提高气体治疗效果， 还可以极大地避免药物对正常组织的毒副作用。

\section{4 基于治疗性气体的多模式协同治疗}

多模式协同治疗是将多种治疗模式联合，达到 联合增效的作用。不同于其它常规的多模式联合治 疗, 将气体治疗与其它治疗方式进行联合的多模式 协同治疗不仅可以增强治疗效果，而且治疗性气体 还可以减弱甚至消除其它治疗对正常组织带来的毒 副作用。例如, $\mathrm{CO}$ 气体在增效化疗的同时, 还可以 为化疗解毒 ${ }^{[16]}$ 。这种基于治疗性气体的多模式协同 治疗明显优于传统的多模式协同治疗, 是安全、高 效治疗所迫切期望的一种策略。借助铃铛型介孔二 氧化硅纳米载体和新型二维多功能纳米载体, 可以 以纳米药物形式有机整合气体治疗与其它治疗模式 (如热疗、化疗、放疗、光治疗和免疫治疗等), 进行 多模式联合治疗, 对于提高抗癌效率、降低毒副作 
(A)
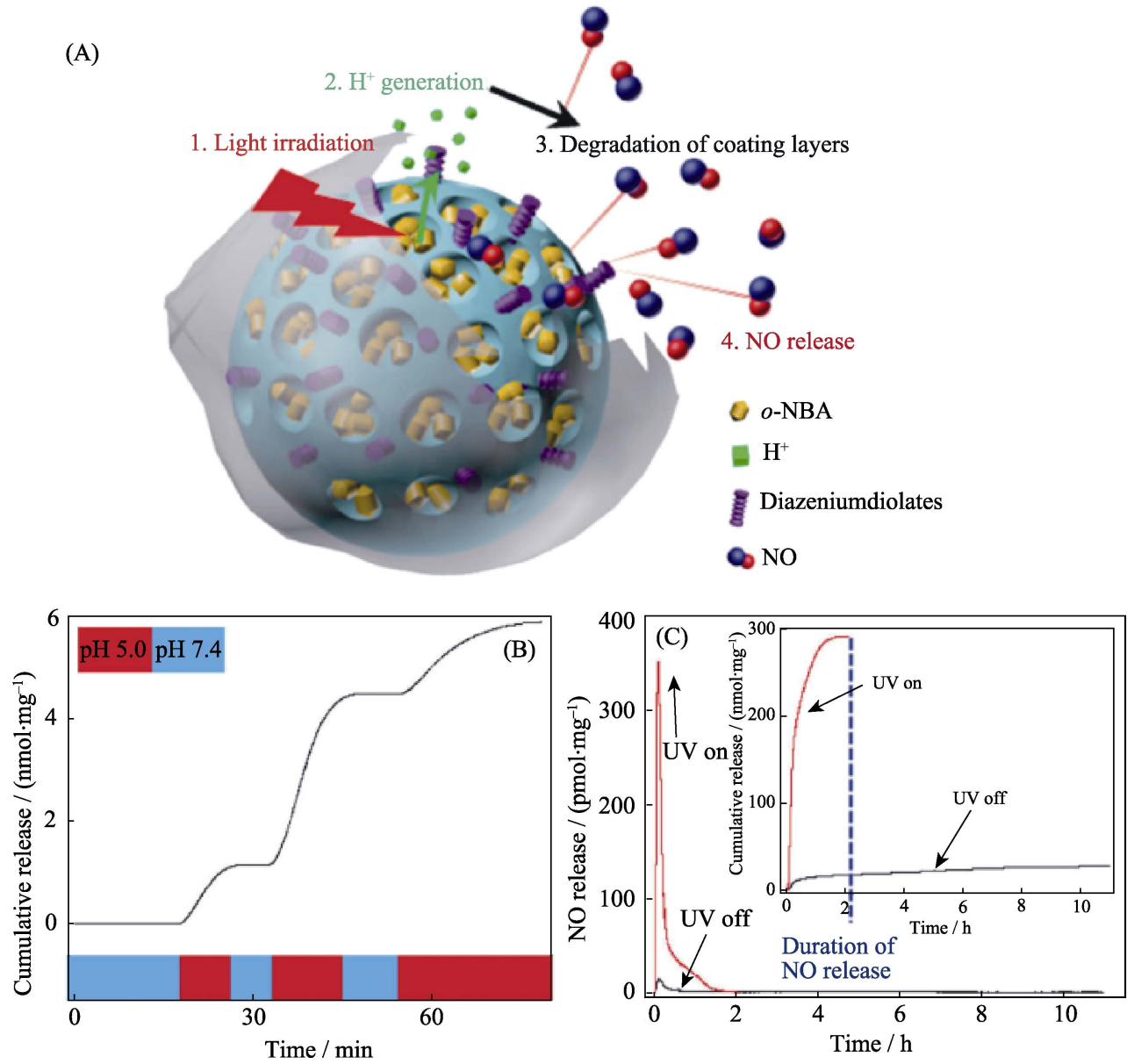

图 8 (A)通过介孔二氧化硅纳米载体包裹 $\mathrm{NO}$ 气体前药并涂覆磷酸钲响应层构建的新型纳米药物 MSN-CaP-NO 及其控释原理示意图, (B)纳米药物的酸响应 NO 释放行为和(C)纳米药物的光控 NO 释放行为 ${ }^{[32]}$

Fig. 8 (A) The construction of the MSN-CaP-NO nanomedicine and its controlled NO release mechanism,

(B) acid-responsive NO release behavior of the nanomedicine, and (C) light-controlled NO release profile of the nanomedicine ${ }^{[32]}$ MSN: Mesoporous Silica Nanoparticles

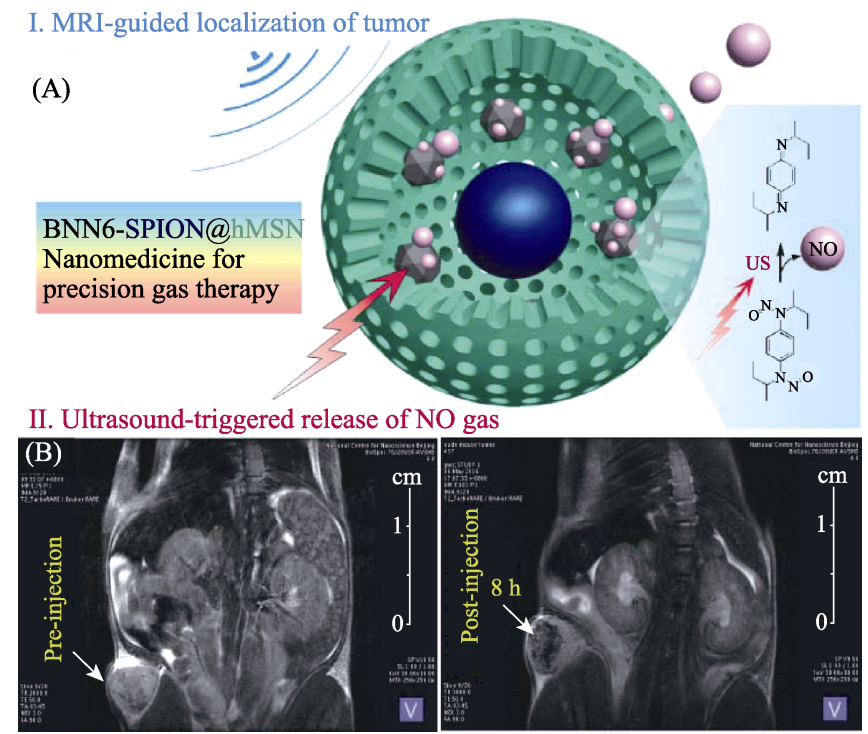

图 9 (A)具有“铃铛”结构的 BNN6-SPION@hMSN 纳米药物的 MRI 成像引导超声可控 NO 气体释放的原理示意图, 和(B)纳米药物的肿瘤靶向行为和 MRI 成像照片 ${ }^{[41]}$

Fig. 9 (A) The MRI-guided ultrasound-triggered NO release mechanism of BNN6-SPION@hMSN nanomedicine, and

(B) tumor-targeting property and the corresponding MRI profile $\mathrm{e}^{[41]}$

BNN: bis- $N$-nitroso; SPION: Superparamagnetic Iron Oxide-encapsulated; hMSN: hollow Mesoporous Silica Nanoparticles 

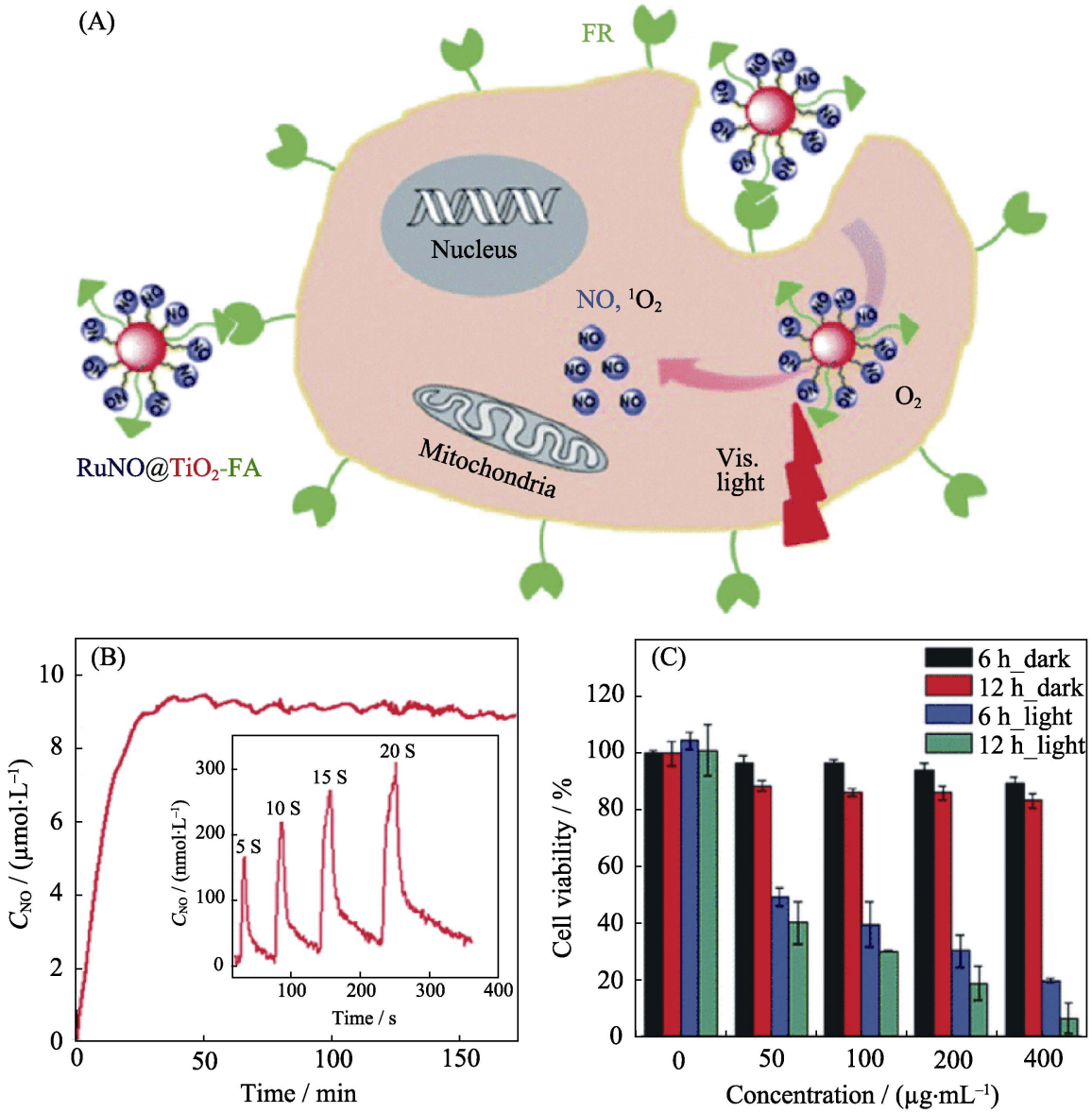

图 10 (A) 由二氧化钣纳米粒包裹 $\mathrm{NO}$ 前药构建的纳米药物 $\mathrm{RuNO} @ \mathrm{TiO}_{2} \mathrm{NPs}$ 及其光控 $\mathrm{ROS}$ 和 $\mathrm{NO}$ 共释放原理示意图, (B)纳米药物的光控 $\mathrm{NO}$ 释放行为和 $(\mathrm{C})$ 纳米药物的细胞毒性研究 ${ }^{[48]}$

Fig. 10 (A) The construction of the RuNO@ $0 \mathrm{TiO}_{2} \mathrm{NPs}_{\mathrm{N}}$ nanomedicine and its light-controlled ROS/NO co-release mechanism, (B) behavior of light-responsive release of $\mathrm{NO}$ and (C) cytotoxicity profile of the nanmedicine ${ }^{[48]}$

用具有重要的科学意义 ${ }^{[19]}$ 。借助多功能纳米载体的 成像性质，还可以将基于气体的多模式治疗与多模 式成像有机结合，对于实现高效的精准诊疗具有重 要的科学意义。

在联合肿瘤化疗、放疗和光动力学治疗中, $\mathrm{CO}$ 和 $\mathrm{NO}$ 等治疗性气体既可以特异性地敏化肿瘤细胞 也可以保护正常细胞。文献[48]通过构建一种亚硝 基钉配合物包裹二氧化钛纳米晶的核壳纳米结构 (图 10(A)), 实现了光响应下 $\mathrm{NO}$ 和单线态氧共释放 (图 10(B)), 成功地在一个复合纳米颗粒上进行气体 治疗和光动力学治疗, 展现了协同增强的抗肿瘤效 果 (图 10(C))。最近, 本课题组 ${ }^{[31]}$ 采用柔性 mPEG-PLGA 载体共装载光响应性 BNN6 前药和化 疗药物阿霉素, 构建了一种新型多功能纳米气体药 物(图 11(A))。该纳米药物在光刺激作用下释放 NO 气体, 导致 mPEG-PLGA 载体的破裂(图 11(B)), 从 而引起 DOX 药物的共释放, 实现气体治疗和化疗 的联合治疗(图 11(C)和(D))。此外, 本课题组还借助 空心介孔二氧化硅纳米载体, 构建了多种新型纳米
气体药物(图 5 和图 7), 实现 $\mathrm{X}$ 射线和葡萄糖响应性 控释 $\mathrm{NO}$ 气体的同时, 将 $\mathrm{NO}$ 气体治疗与放疗和饥 饿疗法进行了有机整合(图 7(C)), 实现了气体治疗 与放疗和饥饿治疗联合增效的目的。除了化疗、放 疗和光动力学治疗, 其他治疗模式(如热疗和免疫 治疗等)也有望借助多功能纳米载体与气体治疗进 行多模式整合，实现高效低毒的精准气体治疗。

\section{5 总结与展望}

气体治疗是一种新兴的、且非常有应用前景的 抗癌治疗策略。将纳米技术应用于气体治疗领域将 产生深远的影响。纳米气体治疗着重解决可控气体 释放和靶向气体运输两个关键科学问题。通过内源 性和外源性刺激源控制气体释放的研究方兴未艾, 还有很多值得进一步开发之处，譬如内源性酶响应 性可控气体释放和外源性磁响应性可控气体释放 等。靶向气体传输的研究还非常欠缺, 但却至关重 要, 应当引起足够的重视, 开展更多的研究。气体治 
(A)
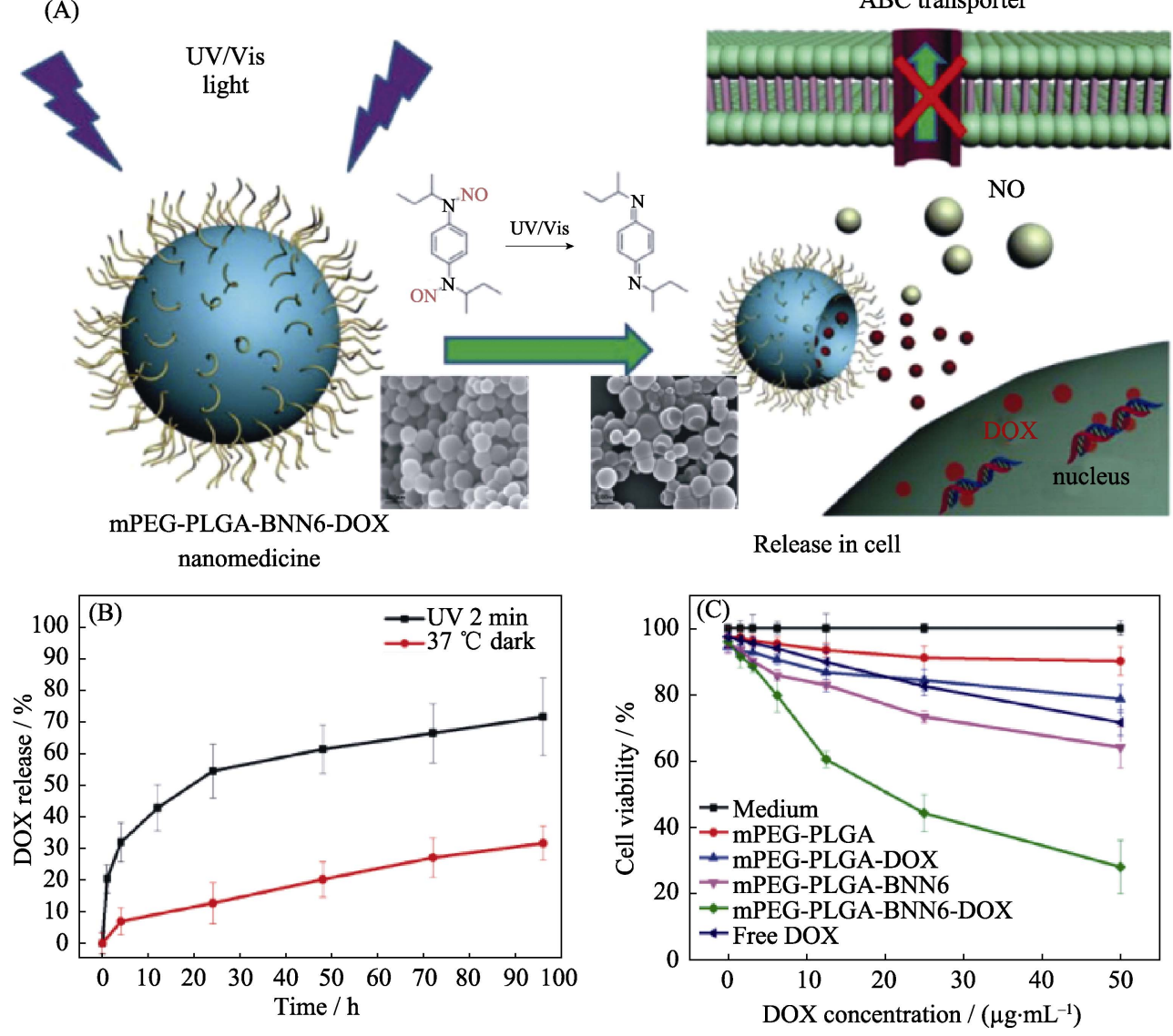

图 11 (A)mPEG-PLGA 载体共担载化疗药物 DOX 和气体前药 BNN6 构建的纳米药物的光控多药共释原理示意图, (B)纳米药物的光控释放 NO 行为和(C)纳米药物的细胞毒性研究 ${ }^{[31]}$

Fig. 11 (A) The mechanism of light-controlled multidrug co-release from the mPEG-BNN6-DOX nanomedicine, (B) behavior of UV-controlled NO release of the nanomedicine, and (C) cytotobxicity profile of the nanomedicine ${ }^{[31]}$ BNN: bis- $N$-nitroso; DOX: Doxorubicin

疗的体内监控是保证治疗安全性的一个重要方面, 但相关研究还仅仅局限于体外水平和细胞层面 ${ }^{[49-53]}$, 动物体内水平的研究还亟待开展, 这主要受限于先 进纳米气体诊疗体系设计的困难性。

\section{参考文献:}

[1] SZABÓ C. Gasotransmitters in cancer: from pathophysiology to experimental therapy. Nat. Rev. Drug Discov., 2016, 15(3): 185203.

[2] SZABÓ C. Hydrogen sulphide and its therapeutic potential. Nat. Rev. Drug Discov., 2007, 6(11): 917-935.

[3] FUKUMURA D, KASHIWAGI S, JAIN R K. The role of nitric oxide in tumour progression. Nat. Rev. Cancer., 2006, 6(7): 521534.

[4] MOTTERLINI R, OTTERBEIN L E. The therapeutic potential of carbon monoxide. Nat. Rev. Drug. Discov., 2010, 9(9): 728-743.

[5] GALLEGO S G, BERNARDES G J L. Carbon-monoxide-releasing molecules for the delivery of therapeutic $\mathrm{CO}$ in vivo. Angew. Chem. Int. Ed., 2014, 53(37): 9712-9721.
[6] BIBHUTI B M, VIJAY A K R, GREGORY W M, et al. Nitric oxide controls the immunopathology of tuberculosis by inhibiting NLRP3 inflammasome-dependent processing of IL-1 $\beta$ Christian Bogdan. Nat. Immunol., 2013, 14(1): 52-60.

[7] LALA P K, CHAKRABORTY C. Role of nitric oxide in carcinogenesis and tumour progression. Lancet Oncol., 2001, 2(3): 149156.

[8] CHIN B Y, JIANG G, WEGIEL B, et al. Hypoxia-inducible factor 1alpha stabilization by carbon monoxide results in cytoprotective preconditioning. Proc. Natl. Acad. Sci. USA, 2007, 104(12): 5109-5114.

[9] OTTERBEIN L E, ZUCKERBRAUN B S, HAGA M, et al. Carbon monoxide suppresses arteriosclerotic lesions associated with chronic graft rejection and with balloon injury. Nat. Med., 2003, 9(2): 183-190.

[10] OTTERBEIN L E, BACH F H, ALAM J, et al. Carbon monoxide has anti-inflammatory effects involving the mitogen-activated protein kinase pathway. Nat. Med., 2000, 6(4): 422-428.

[11] NASSOUR I, KAUTZA B, RUBIN M, et al. Carbon monoxide protects against hemorrhagic shock and resuscitation-induced microcirculatory injury and tissue injury. Shock, 2015, 43(2): 166- 
171.

[12] CARPENTER A W, SCHOENFISCH M H. Nitric oxide release: Part II. Therapeutic applications. Chem. Soc. Rev., 2012, 41(10): $3742-3752$.

[13] DIRING S, WANG D O, KIM C, et al. Localized cell stimulation by nitric oxide using a photoactive porous coordination polymer platform. Nat. Commun., 2013, 4(10): 2684.

[14] KIM J, SARAVANAKUMAR G, CHOI H W, et al. A platform for nitric oxide delivery. J. Mater. Chem., 2014, 2(4): 341-356.

[15] RAJU T N. The Nobel chronicles. 1998: Robert Francis Furchgott (b 1911), Louis J Ignarro (b 1941), and Ferid Murad (b 1936). Lancet, 2000, 356(9226): 346.

[16] WEGIEL B, GALlo D, CSIZMADIA E, et al. Carbon monoxide expedites metabolic exhaustion to inhibit tumor growth. Cancer Res., 2013, 73(23): 7009-7021.

[17] MONCADA S, ERUSALIMSKY J D. Does nitric oxide modulate mitochondrial energy generation and apoptosis? Nat. Rev. Mol. Cell Biol., 2002, 3(4): 214-220.

[18] MÓDIS K, BOS E M, CALZIA E, et al. Regulation of mitochondrial bioenergetic function by hydrogen sulfide. Part II. Pathophysiological and therapeutic aspects. Br. J. Pharmacol., 2014, 171(8): 2123-2146.

[19] HE Q J. Precision gas therapy using intelligent nanomedicine. Biomater. Sci., 2017, 5(11): 2226-2230.

[20] ERNST A, ZIBRAK J D. Carbon monoxide poisoning. N. Engl. J. Med., 1998, 339(22): 1603-1608.

[21] SULLIVAN J B, KRIEGER G B, THOMAS R J. Methemoglobin-forming chemicals in hazardous materials toxicology: clinical principals of environmental health. J. Occup. Environ. Med., 1992, 34(4): $365-371$.

[22] RIDNOUR L A, THOMAS D D, DONZELLI S, et al. The biphasic nature of nitric oxide responses in tumor biology. Antioxid. Redox. Sign., 2006, 8(7/8): 1329-1337.

[23] WANG L Z, SHI J L, YUN J, et al. Research progress of mesoporous silicon materials. J. Inorg. Mater., 1999, 14(3): 333-342.

[24] SHI J L, CHEN Y, CHEN H R. Research progress of multifunctional mesoporous silica based nanoparticles for diagnosis and treatment. J. Inorg. Mater., 2013, 28(1): 1-11.

[25] HE Q J, SHI J L. MSN anti-cancer nanomedicines: chemotherapy enhancement, overcoming of drug resistance and metastasis inhibition. Adv. Mater., 2014, 26(3): 391-411.

[26] ZHOU Y L, FENG X X, ZHAI W Y. Research on loading and release of Epirubicin with mesoporous bioactive glass. J. Inorg. Mater., 2011, 26(1): 68-72.

[27] HE Q, KIESEWETTER D O, QU Y, et al. NIR-responsive on-demand release of $\mathrm{CO}$ from metal carbonyl-caged graphene oxide nanomedicine. Adv. Mater., 2015, 27(42): 6741-6746.

[28] FAN J, HE N Y, HE Q J, et al. A novel self-assembled sandwich nanomedicine for NIR-responsive release of NO. Nanoscale, 2015, 7(47): 20055-20062.

[29] GARCIA J V, YANG J, SHEN D, et al. NIR-triggered release of caged nitric oxide using upconverting nanostructured materials. Small, 2012, 8(24): 3800-3805.

[30] ZHANG X, TIAN G, YIN W, et al. Controllable generation of nitric oxide by near-infrared-sensitized upconversion nanoparticles for tumor therapy. Adv. Funct. Mater., 2015, 25(20): 3049-3055.

[31] FAN J, HE Q, LIU Y, et al. Light-responsive biodegradable nanomedicine overcomes multidrug resistance via NO-enhanced chemosensitization. ACS Appl. Mater. Interfaces, 2016, 8(22): 13804-1381.

[32] WOO C H, JIHOON K, JINHWAN K, et al. Light-induced acid generation on a gatekeeper for smart nitric oxide delivery. ACS Nano, 2016, 10(4): 4199-4208.

[33] OSTROWSKI D, LIN B F, TIRRELL M V, et al. Liposome encapsulation of a photochemical NO precursor for controlled nitric oxide release and simultaneous fluorescence imaging. Mol. Pharm., 2012, 9(10): 2950-2955.

[34] WANG P G, XIAN M, TANG X P, et al. Nitric oxide donors: Chemical activities and biological applications. Chem. Rev., 2002, 102(4): 1091-1134.

[35] RIMMER R D, PIERRI A E, FORD P C. Photochemically activated carbon monoxide release for biological targets. toward developing air-stable photoCORMs labilized by visible light. Coordin. Chem. Rev., 2012, 256(15/16): 1509-1519.

[36] ZHENG D W, LI B, LI C X, et al. Photocatalyzing $\mathrm{CO}_{2}$ to $\mathrm{CO}$ for enhanced cancer therapy. Adv. Mater., 2017, 29(44): 1703822-1-8.

[37] Reddy G U, AXTHELM J, HOFFMANN P, et al. Co-registered molecular logic gate with a CO-releasing molecule triggered by light and peroxide. J. Am. Chem. Soc., 2017, 139(14): 4991-4994.

[38] CHEN L J, HE Q J, LEI M Y, et al. Facile coordination-precipitation route to insoluble metal roussin's black salts for NIR-responsive release of NO for anti-metastasis. ACS Appl. Mater. Interfaces., 2017, 9(42): 36473-36477.

[39] MARIN A, MUNIRUZZAMAN M, RAPOPORT N. Mechanism of the ultrasonic activation of micellar drug delivery. J. Control. Release, 2001, 75(1/2): 69-81.

[40] POSTEMA M, BOUAKAZ A, CATE F J T, et al. Nitric oxide delivery by ultrasonic cracking: some limitations. Ultrasonics, 2006, 44(Suppl.): e109-e113.

[41] JIN Z, WEN Y, HU Y, et al. MRI-guided and ultrasound-triggered release of NO by advanced nano-medicine. Nanoscale, 2017, 9(10): $3637-3645$.

[42] FAN W, BU W, HE Q, et al. X-ray radiation-controlled NO-release for on-demand depth-independent hypoxic radiosensitization. Angew. Chem. Int. Ed., 2015, 54(47): 14026-14030.

[43] JIN Z, WEN Y, XIONG L, et al. Intratumoral $\mathrm{H}_{2} \mathrm{O}_{2}$-triggered release of $\mathrm{CO}$ from a metal carbonyl-based nanomedicine for effi- 
cient CO therapy. Chem. Commun., 2017, 53(40): 5557-5560.

[44] FAN W, LU N, HUANG P, et al. Glucose-responsive sequential generation of hydrogen peroxide and nitric oxide for synergistic cancer starving-like/gas therapy. Angew. Chem. Int. Ed., 2016, 55(1): $1-6$.

[45] HE Q J, GUO S R, QIAN Z Y, et al. Development of individualized anti-metastasis strategies by engineering nanomedicines. Chem. Soc. Rev., 2015, 44(17): 6258-6286.

[46] GUI R J, WAN A J, ZHANG Y L, et al. Light-triggered nitric oxide release and targeted fluorescence imaging in tumor cells developed from folic acid-graft-carboxymethyl chitosan nanospheres. RSC Adv., 2014, 4(57): 30129-30136.

[47] ZHANG X F, MANSOURI S, MBEH D A, et al. Nitric oxide delivery by core/shell superparamagnetic nanoparticle vehicles with enhanced biocompatibility. Langmuir, 2012, 28(35): 12879-12885.

[48] XIANG J, AN L, TANG W W, et al. Photo-controlled targeted intracellular delivery of both nitric oxide and singlet oxygen using a fluorescencence trackable ruthenium nitrosyl functional nanoplatform. Chem. Commun., 2015, 51(13): 2555-2558.

[49] CHAKRABORTY I, JIMENEZ J, SAMEERA W M, et al. Lumi- nescent Re(I) carbonyl complexes as trackable PhotoCORMs for CO delivery to cellular targets. Inorg. Chem., 2017, 56(5): 28632873.

[50] JI X, ZHOU C, JI K, et al. Click and release: a chemical strategy toward developing gasotransmitter prodrugs by using an intramolecular Diels-Alder reaction. Angew. Chem. Int. Ed., 2016, 55(1): $1-7$.

[51] CARRINGTON S J, CHAKRABORTY I, BERNARD J M L, et al. A theranostic two-tone luminescent PhotoCORM derived from $\operatorname{Re}(\mathrm{I})$ and (2-pyridyl)-benzothiazole: trackable CO delivery to malignant cells. Inorg. Chem., 2016, 55(16): 7852-7858.

[52] CHAKRABORTY I, CARRINGTON S J, HAUSER J, et al. Rapid eradication of human breast cancer cells through trackable lighttriggered $\mathrm{CO}$ delivery by mesoporous silica nanoparticles packed with a designed photoCORM. Chem. Mater., 2015, 27(24): 83878397.

[53] CARRINGTON S, CHAKRABORTY I, BERNARD J M L, et al. Synthesis and characterization of a "turn-on" photoCORM for trackable CO delivery to biological targets. ACS Med. Chem. Lett., 2014, 5(12): 1324-1328. 